\title{
Psychological parameters have units: A bug fix for stochastic prospect theory and other decision models
}

\author{
Neil Stewart \\ University of Warwick \\ Benjamin Scheibehenne \\ University of Geneva \\ Thorsten Pachur \\ Max Planck Institute for Human Development
}

\begin{abstract}
To fit models like prospect theory or expected utility theory to choice data, a stochastic model is needed to turn differences in values into choice probabilities. In these models, the parameter measuring risk aversion is strongly correlated with the parameter measuring the sensitivity to differences in value. We use dimensional analysis from the physical sciences to show that this is because the sensitivity parameter has units which depend on the risk aversion parameter. This means that comparing sensitivities across individuals with different level of risk aversion is meaningless and forbidden. We suggest a simple bug fix for prospect theory and other decision models which corrects this problem. The bug fix completely removes the correlation between sensitivity and risk aversion parameters in model estimations and allows the parameters to be interpreted as they were originally intended.
\end{abstract}

Keywords: dimensional analysis, cumulative prospect theory, risk aversion, sensitivity

Fourier (1822) presented the concept of physical dimension, which relates physical quantities (e.g., distance or time) and their units (e.g., in the metric system, meters and seconds). Psychological quantities have units as well, and it is this observation that leads

April 10, 2018

Stewart, N., Scheibehenne, B., \& Pachur, T. (2018). Psychological parameters have units: A bug fix for stochastic prospect theory and other decision models. Manuscript submitted for publication.

Correspondence concerning this article should be addressed to Neil Stewart, Warwick Business School, University of Warwick, Coventry, CV4 7AL, England. E-mail: neil.stewart@wbs.ac.uk

This work was supported Economic and Social Research Council grants ES/K002201/1, ES/K004948/1, ES/N018192/1, and Leverhulme grant RP2012-V-022.

$\mathrm{R}$ code for the simulations is available from the authors.

We thank Lukasz Walasek and Timothy L. Mullett for discussion. 
us to suggest a change in the implementation of stochastic versions of prospect theory and other decision models. Our suggested change eliminates the substantial correlations between model parameter values found in fits to choice data.

\section{The Intuition}

Consider choosing between Gamble A offering a "sure \$100" and Gamble B offering "a $50 \%$ chance of $\$ 200$ otherwise nothing". In many theories, the dollar amounts are translated into psychological values by a value function $v(\cdot)$, which is often implemented as a simple power function $v(x)=x^{\alpha}$, where the utility of $\$ \mathrm{x}$ is $x^{\alpha}$. Consider setting $\alpha=\frac{1}{2}$. The expected psychological value of Gamble A is higher than the expected psychological value of Gamble B:

$$
V(A)-V(B)=v(\$ 100)-0.5 \times v(\$ 200)=100^{\frac{1}{2}}-0.5 \times 200^{\frac{1}{2}}=10.00-7.07=2.93>0
$$

The difference in expected psychological value is greater than zero, so we say the decision maker prefers Gamble A. We say the decision maker is risk averse because he or she prefers the sure Gamble A to the risky Gamble B, despite the gambles having equal expected value, both paying out an average of $\$ 100$.

Now consider setting $\alpha=2$. The expected psychological value of Gamble A is now lower than the expected psychological value of Gamble B.

$V(A)-V(B)=v(\$ 100)-0.5 \times v(\$ 200)=100^{2}-0.5 \times 200^{2}=10000-20000=-10000<0$

The difference is less than zero, so we say the decision maker prefers Gamble B. We say the decision maker is risk seeing because he or she prefers the risky Gamble B, despite the gambles having equal expected value.

Key to the intuition is to notice the very different magnitudes of the differences in expected psychological value when $\alpha$ changes. When $\alpha=\frac{1}{2}$ we had a difference of 2.93 . When $\alpha=2$ we had a difference of -10000.00 . One difference is over 1000 times larger in magnitude than the other. We will return to this observation below.

In stochastic choice models, the difference in psychological values is often translated into a choice probability using the logistic choice rule in Equation 1.

$$
p(A, B)=\frac{1}{1+e^{-\theta[V(A)-V(B)]}}
$$

The sensitivity parameter $\theta$ controls how fast choice probabilities change with differences in psychological value. Figure 1 shows how a difference in psychological value (which can be any real value, positive or negative) is translated into a choice probability (any real number in the interval 0-1). For example, below we will consider how, for $\alpha=\frac{1}{2}$, the difference in psychological value of 2.93 favoring Gamble A is translated into a .7 probability of choosing Gamble A.

If large differences in psychological value are to be converted into sensible choice probabilities, then $\theta$ will need to be a small number. If small differences in psychological value are to be converted into sensible choice probabilities, then $\theta$ will need to be a much larger number. For example, if our risk averse decision maker with $\alpha=\frac{1}{2}$ prefers the 


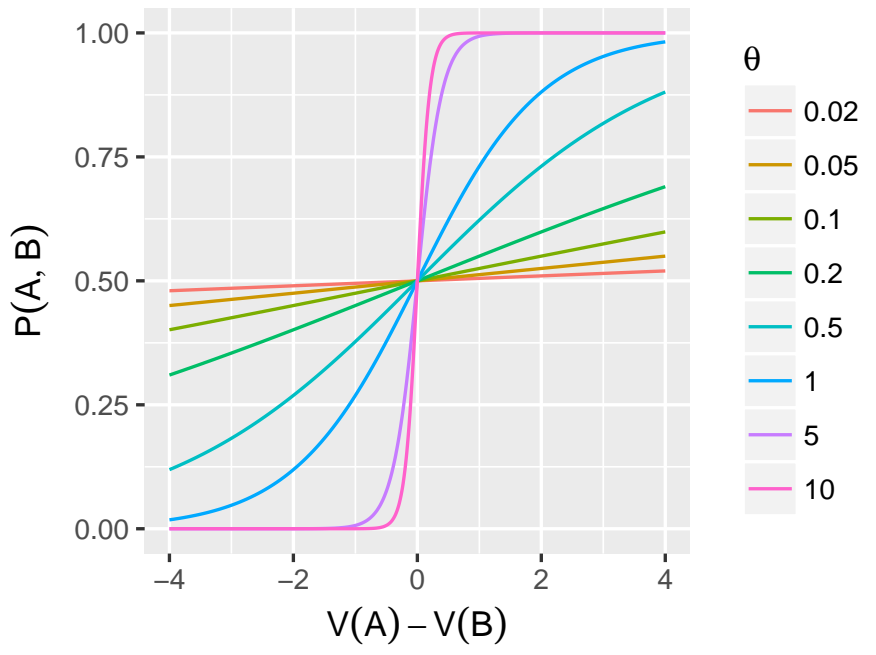

Figure 1. The logistic function.

sure Gamble A $70 \%$ of the time, the $\theta$ must take a value of 0.29 . But if our risk seeking decision maker with $\alpha=2$ prefers the sure Gamble B 70\% of the time (i.e., so the strength of preference for the risky option for the risk-seeking decision maker is as strong as the strength of preference for the safe option for the risk-averse decision maker), $\theta$ must take a value of 0.000084 . Our $\theta$ estimates are several orders of magnitude different because the differences in psychological value are several orders of magnitude different. Basically, to convert very different magnitude differences in psychological value into moderate choice probabilities, one needs $\theta$ to have very different magnitudes too.

Fourier's concept of physical dimension helps make clear what is going on here. When $\alpha=\frac{1}{2}$, the units of psychological value are $\$ \frac{1}{2}$ - square root dollars. When $\alpha=2$, the units of psychological value are $\$^{2}$-dollars squared. More generally, the units of psychological value for any $\alpha$ are $\$^{\alpha}$. This means that our psychological differences of 2.09 and -10000 are simply incommensurable. The 2.09 has units of $\$ \frac{1}{2}$. The -10000 has units of $\$^{2}$. We cannot compare them, just as we cannot compare a weight of $2.09 \mathrm{~kg}$ to distance of $10000 \mathrm{~m}$. Numbers on different dimensions are incommensurable.

Another consequence of Fourier's dimensional analysis concerns $\theta$. Psychological values can have units, and $\theta$ generally does have units. A consequence of dimensional analysis is that any power must be dimensionless. For example, $x^{2}$ means $x \times x$. The 2 is a dimensionless real number which describes the number of times $x$ is multiplied by itself. Applied to Equation 1, this means that the power of $e$, which is $-\theta \times[V(A)-V(B)]$ must be dimensionless. This means that $\theta$ must have the inverse of whatever the unit of $[V(A)-V(B)]$ is, so that the units cancel in the multiplication. So when $\alpha=\frac{1}{2}$, and psychological values and their differences have units of $\$^{\frac{1}{2}}$ or $\sqrt{\$}$, then $\theta$ must have units of per $\sqrt{\$}$ or $\$^{-1 / 2}$. 
Our $\theta$ parameters of 0.29 is 0.29 per $\sqrt{\$}$ or $0.29 \$^{-1 / 2}$. And when $\alpha=2$ and psychological values and their differences have units of $\$^{2}$, then $\theta$ must have units of per $\$^{2}$ or $\$^{-2}$. Our $\theta$ parameter of 0.000084 is 0.000084 per $\$^{2}$ or $0.000084 \$^{-2}$. In general, $\theta$ has units of $\$^{-\alpha}$.

This is a disaster - one parameter has units that depend on the other. What does this mean? We know that quantities with different units are incommensurable. In this case, this means that comparisons of $\theta$ across individuals with different $\alpha$ are meaningless. To the extent that comparisons of $\theta$ are meaningless when $\alpha$ is varying, one should never plot a histogram of $\theta \mathrm{s}$, or a scatter plot with $\theta$. Practically, this means that $\alpha$ and $\theta$ are also going to be negatively correlated. This is because a large $\alpha$ means that the psychological values and their differences will be large numbers and that a small $\theta$ is thus required to scale them down for transformation through the logistic function. And a small $\alpha$ means that the psychological values and their differences will be small numbers and that a large $\theta$ is required to scale them up for transformation through the logistic function. The negative correlation between $\theta$ and $\alpha$ is a big problem when trying to recover prospect theory parameters from choice data. The remainder of this paper highlights this problem and presents a bug fix to correct the problem.

\section{A Bug Fix for Cumulative Prospect Theory}

\section{Proof of Concept in Likelihood Surfaces}

In cumulative prospect theory (CPT, Tversky \& Kahneman, 1992), each risky gamble is assigned a deterministic subjective value. Stott (2006) reviews the possible functional forms that can be used in CPT. We have used the forms from Tversky and Kahneman's original presentation. Consider a gamble $G=\left(q_{n}, y_{n} ; q_{n-1}, y_{n-1} ; \ldots ; p_{m-1}, x_{m-1}, p_{m}, x_{m}\right)$ where $p_{i}$ is the probability of gain $x_{i}, q_{i}$ is the probability of loss $y_{i} . m$ indexes the gains, and $n$ indexes the losses, with the $x_{i}$ s are ordered so $i=n$ for the worst outcome and $i=n-1$ for the second worst, through to $i=m-1$ for the second best and $i=m$ for the best. Equation 2 shows how the psychological value is a sum of values for the gains and losses parts of the gamble.

$$
V(G)=\sum_{i=1}^{n} v\left(y_{i}\right) \pi_{i}^{-}+\sum_{i=1}^{m} v\left(x_{i}\right) \pi_{i}^{+}
$$

The value function $v(\cdot)$ transforms outcomes into psychological value, and is defined in Equation 3 which has power $\alpha$ controlling the curvature for gains, power $\beta$ controlling the curvature for losses, and loss aversion parameter $\lambda$ representing the extent to which losses loom larger than gains.

$$
\begin{array}{ll}
v(x)=x^{\alpha} & x \geq 0 \\
v(y)=-\lambda|y|^{\beta} & y<0
\end{array}
$$

We have set $\beta=\alpha$ in our application (see Discussion; Nilsson, Rieskamp, \& Wagenmakers, 2011). The $\pi_{i}$ s from Equation 2 are decision weights for each branch, and are given by transforming the cumulative probability of receiving at least as much as $x_{i}$ or at least as little as $y_{i}$. Weighting is done separately for the gain and loss parts of the gamble. 


$$
\begin{array}{ll}
\pi_{m}^{+}=w^{+}\left(p_{m}\right) & \\
\pi_{n}^{-}=w^{-}\left(q_{n}\right) & \\
\pi_{i}^{+}=w^{+}\left(p_{i}+\cdots+p_{m}\right)-w^{+}\left(p_{i+1}+\cdots+p_{m}\right) & \text { for } 1 \geq i<m \\
\pi_{j}^{-}=w^{-}\left(q_{j}+\cdots+q_{n}\right)-w^{-}\left(q_{j+1}+\cdots+a_{n}\right) & \text { for } 1 \geq j<n
\end{array}
$$

The weighting functions $w^{+}(\cdot)$ and $w^{-}(\cdot)$ are given in Equation 5, where the $\gamma^{+}$ parameter controls the curvature of the weighting function for gains and $\gamma^{-}$controls the curvature for losses. We have used $\gamma^{+}=\gamma^{-}=\gamma$ in our application, to reduce the possibility that the $\lambda$ and $\gamma^{+}$and $\gamma^{-}$trade off against one another (Pachur \& Kellen, 2013).

$$
\begin{array}{ll}
w^{+}(p) & =\frac{p^{\gamma^{+}}}{\left(p^{\gamma^{+}}+(1-p)^{\gamma^{+}}\right)^{1 / \gamma^{+}}} \text {for gains } \\
w^{-}(q) & =\frac{q^{\gamma^{-}}}{\left(q^{\gamma^{-}}+(1-q)^{\gamma^{-}}\right)^{1 / \gamma^{-}}} \quad \text { for losses }
\end{array}
$$

To make probabilistic predictions for choices between gambles, the psychological values $V(A)$ and $V(B)$ are often transformed into choice probabilities using an exponential choice rule, as recommended by Stott (2006) and used by Rieskamp (2008), Glöckner and Pachur (2012), and Scheibehenne and Pachur (2015), amongst others.

$$
p(A, B)=\frac{e^{-\theta V(A)}}{e^{-\theta V(A)}+e^{\theta V(B)}}
$$

This exponentiated choice rule is just the logistic function from Equation 1. (To see this, divide through the numerator and denominator by $e^{-\theta V(A)}$.) This use of the logistic choice rule also occurs widely in psychology, for example in sequential subjective expected utility (Busemeyer \& Townsend, 1993), and is the widely used softmax rule (Sutton \& Barto, 1998).

To show that this usual stochastic extension of CPT leads to problematic correlations between $\alpha$ and $\theta$, we have plotted out the likelihood of the choice data from Glöckner and Pachur (2012). In their experiment, Glöckner and Pachur had 66 participants make choices between simple gambles, with choices involving only gambles for gains, only gambles for losses, and mixed gambles offering gains and losses. The choice set was made up of 180 randomly generated choices from Rieskamp (2008), 40 choices designed to discriminate between CPT and the priority heuristic from Glöckner and Betsch (2008), 10 choices designed to measure risk aversion from Holt and Laury (2002), and 8 choices designed to measure loss aversion from Gäechter, Johnson, and Herrmann (2007).

The top left panel of Figure 2 plots out the likelihood surface as a function of $\alpha$ and $\theta$, for fixed values of the loss aversion parameter $\lambda=2.25$ and probability weighting parameter $\gamma=0.61$ (the original estimates from Tversky \& Kahneman, 1992). The contours of the surface show a ridge, where $\alpha$ and $\theta$ can trade off against one another without altering the likelihood of the data very much. This correlation is more apparent in the bottom left panel where the surface is replotted on a log-log scale.

And so to the bug fix. We have recalculated the likelihood surface using the modified stochastic form in Equation 7. This form differs from the original only in converting the psychological values for Gambles A and B into the sure amounts of money with the same 


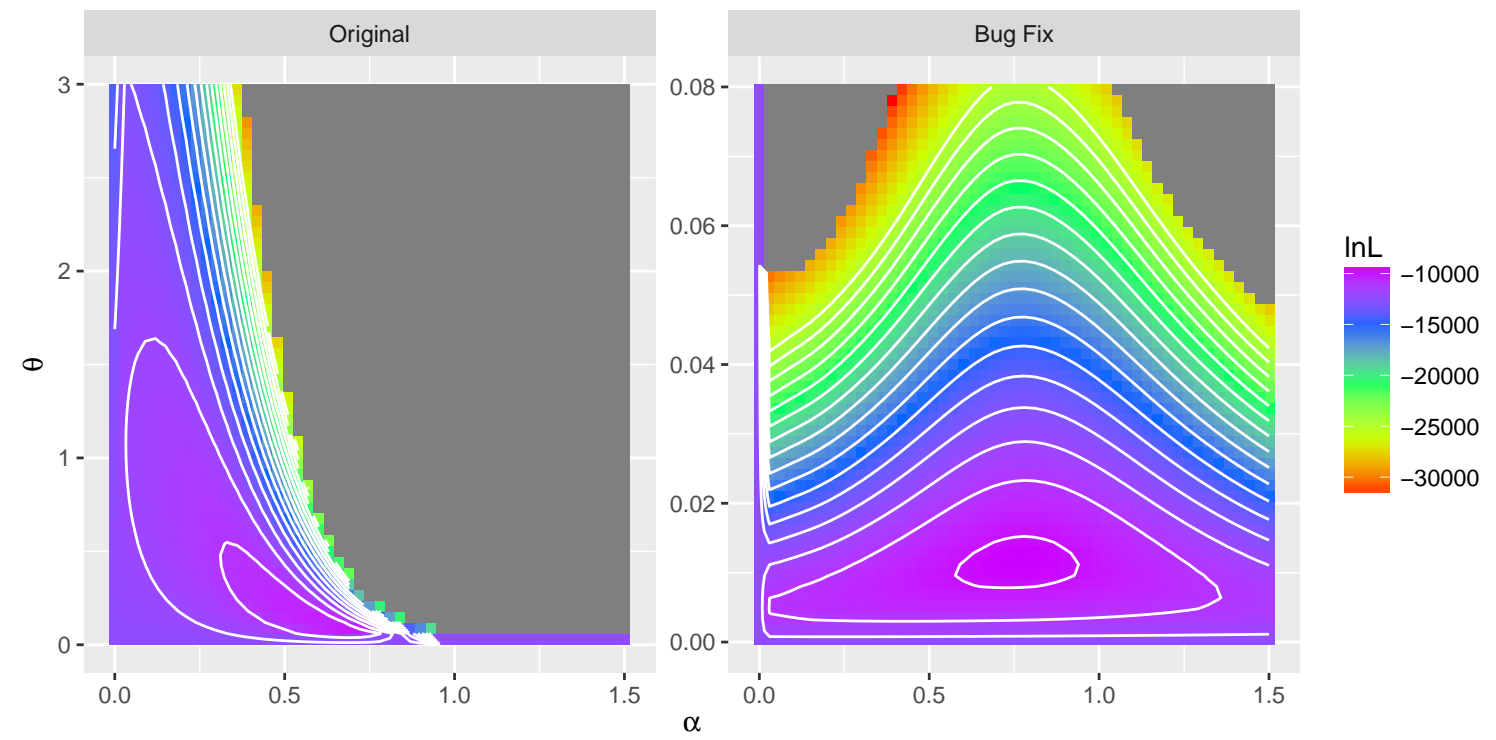

(a) Likelihood in $\alpha-\theta$ space

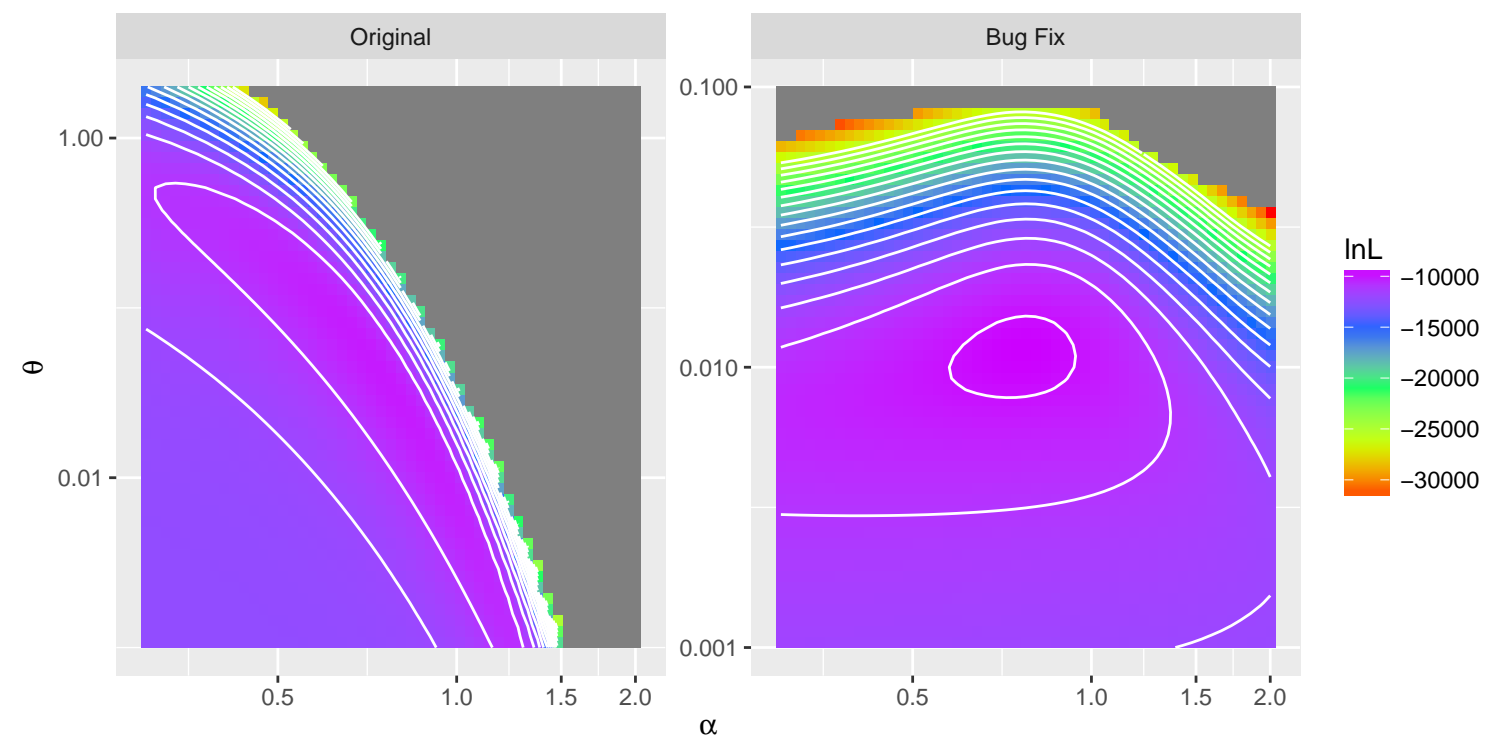

(b) Likelihood in $\log (\alpha)-\log (\theta)$ space

Figure 2. (a) Log likelihood as a function of $\alpha$ and $\theta$ for original prospect theory and the bug fix version with the CE transformation. (b) The same likelihood surfaces, replotted in log-log space. 
psychological value using the inverse value function.

$$
p(A, B)=\frac{1}{1+e^{-\theta\left[v^{-1}(V(A))-v^{-1}(V(B))\right]}}
$$

The inverse value function is the inverse of Equation 3 and is given below in Equation 8 .

$$
\begin{array}{ll}
v^{-1}(z)=z^{1 / \alpha} & \text { for } z \geq 0 \\
v^{-1}(z)=-\left(\frac{|z|}{\lambda}\right)^{1 / \beta} & \text { for } z<0
\end{array}
$$

The bug fix of converting psychological values $z$ into their sure $\$$ equivalents means that the difference always has units of $\$$, which means $\theta$ always has units of per $\$$, or $\$^{-1}$. Now the unit of $\theta$ is free of its dependency on $\alpha$.

The right panels in Figure 2 show the likelihood surfaces for this modified stochastic form. The correlation between $\alpha$ and $\theta$ so apparent in the likelihood surface for the original implementation of CPT is completely eliminated.

In our modified stochastic form, new things are possible. Because each $\theta$ has units of $\$^{-1}$, estimates of $\theta$ are comparable, and it is permitted to plot them in the same figure. It also makes sense to talk of one person being so much more deterministic than the other because one value of $\theta$ is so much higher than another $\theta$. Before the bug fix, when the units of $\theta$ depended on $\alpha$, this makes no sense. In interpreting $\theta$ values in the original formulation, researchers have done this (e.g., Stewart, Reimers, \& Harris, 2015), but it is a mistake.

\section{Illustration with a Bayesian Parameter Recovery}

Scheibehenne and Pachur (2015) presented a Bayesian estimation of CPT parameters for the Glöckner and Pachur (2012) data set. Scheibehenne and Pachur estimated the group level parameters, and we have replicated the hierarchically estimated posterior distribution for these parameters in Figure 3a. (It differs subtly from the original because we extended the bounds on parameters to be $0-5$, and we swapped a two-parameter weighting function for the one-parameter function in Equation 5, and fixed $\gamma^{+}=\gamma^{-}$for simplicity, though the results hold with separate weighting for gains and losses). There is a strong negative association between $\alpha$ and $\theta$, as shown in the top right panel of Figure 3a. As we described above, strictly speaking we should not even be plotting $\theta$ s for different $\alpha$ s on the same plot, as they are incommensurable. Figure 3b shows how this correlation is completely eliminated when the CPT bug fix is introduced. This new estimation followed, in all details, Scheibehenne and Pachur's original estimation, apart from the bug fix.

\section{Discussion}

In applying dimensional analysis to CPT, we have revealed why risk aversion and response sensitivity parameters are correlated and have suggested a modification to the traditional stochastic extension that fixes this problem. The problem was a particularly extreme one, because in the original implementation response sensitivity parameter $\theta$ had units of $\$$ s raised to the power of risk aversion parameter $\alpha$. This means that even small changes in $\alpha$ have huge knock on effects for the units of $\theta$, and thus huge effects of the value of the $\theta$ parameter. Our dimensional analysis has shown that, without our bug fix, 


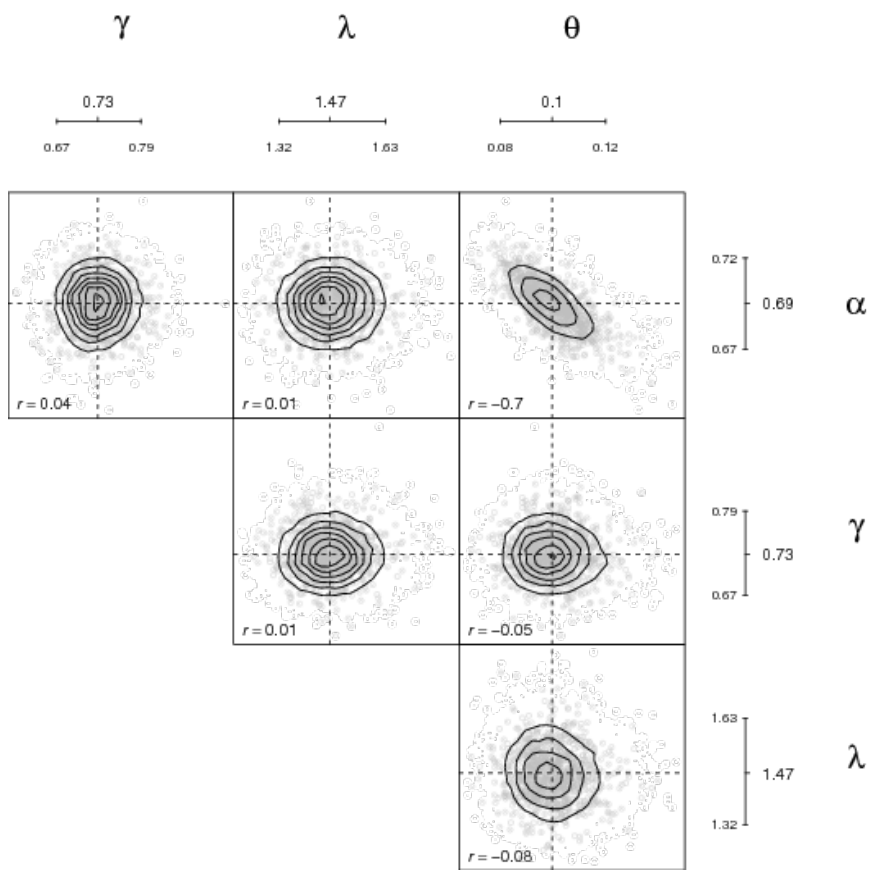

(a) Original CPT

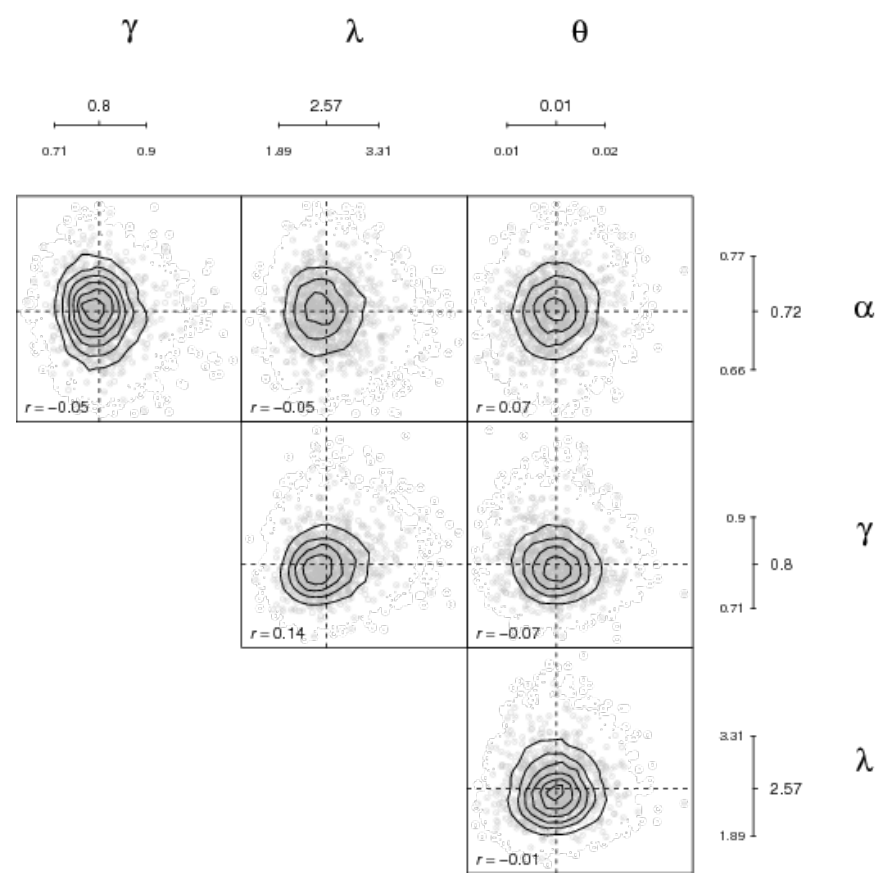

(b) Bug-fix CPT

Figure 3. Group level parameter estimates for fits to data from Glöckner and Pachur (2012) (a) without and (b) with the bug fix. 
one cannot compare $\theta$ estimates when $\alpha$ differs - which it nearly always does. Broomell and Bhatia (2014) mentioned part of the problem we describe here when they said, "Because the units of choice sensitivity are dependent on the units of the gamble payoffs..." (p. 260261) and later, "Outcome scale interacts with choice sensitivity complicating comparisons between decision sets..." (p. 261). What we have done here is to go further and, using concepts from dimensional analysis, point out that the units of $\theta$ incorporate the power $\alpha$.

The bug fix we suggest has been incorporated into boundedly rational expected utility theory (Navarro-Martinez, Loomes, Isoni, \& Butler, 2017). In the theory, utility functions are assumed to fluctuate over the time course of a binary decision, with differences in utility accumulated over time until either an upper or a lower threshold is reached. To allow the utility differences to be summed, they are converted back into certainty equivalentsotherwise, by the method of dimensional analysis, the units for each sampled difference would be different and summing them would not be possible.

The dimensional analysis method also highlights an observation by Wakker (2010) concerning loss aversion. Wakker pointed out that when $\alpha \neq \beta, \lambda$ measures the ratio of psychological values for a gain and loss at 1 unit of money. This means that the meaning of $\lambda$ depends on your choice of units. Is one unit $\$ 1$ or $1 \mathrm{c}$ ? Is one unit $£ 1$ or 1 penny? Given this is arbitrary, just like the choice of miles or kilometres, or kilograms or pounds, this means that the actual value of $\lambda$ is arbitrary too. (See Wakker's Observations 9.6.2 and 9.6.3.) Thinking about what units $\lambda$ must take makes clear why this is true. In CPT the psychological values of gains and losses are summed. This means that they must have the same units. Just as we cannot add 5 meters to 10 seconds because the units are incommensurable, we cannot add $(\$ x)^{\alpha}$ and $(\$ y)^{\beta}$ (unless $\alpha=\beta$ ). And this means that the loss aversion parameter $\lambda$ must have units. Think of $\lambda$ as the exchange rate between the psychological values of gains and losses. If gains are measured in units of $\$^{\alpha}$ and losses are measured in units of $\$^{\beta}$, then $\lambda$ must have units of $\$^{(\alpha-\beta)}$. Effectively the units of $\lambda$ convert loss units into gain units. To see this, consider a sure gain of $\$ x$ being compared to a sure loss of $\$ y$. The sure gain has psychological value of $x^{\alpha}$, which has units of $\$^{\alpha}$. The sure loss has psychological value $\lambda \times y^{\beta}$. This has units of $\$^{(\alpha-\beta)} \times \$^{\beta}$, which reduces to units of $\$^{(\alpha-\beta+\beta)}$ or $\$^{\alpha}$. So the psychological value of the loss $\lambda \times y^{\beta}$ has the same units as the psychological value of the gain $x^{\alpha}$-both have units of $\$^{\alpha}$ and so it is permitted to add them. So $\lambda$ has units, just like physical constants. For example, the constant acceleration due to gravity at the earth's surface has units of metres per second per second in the metric system (and takes the value $9.8 \mathrm{~m} / \mathrm{s}^{2}$ or $9.8 \mathrm{~ms}^{-2}$ ). Under different base units, the value of the acceleration due to gravity is different. For example, if we switch from metres to feet, the acceleration due to gravity is $32.2 \mathrm{ft} / \mathrm{s}^{2}$. Only in the special case when $\alpha=\beta$ is $\lambda$ free of dimensions. This means that $\lambda$ suffers the same problem as $\theta$. It has units that depend on powers of other parameters, and it is meaningless to compare $\lambda$ values across people when $\alpha \neq \beta$. To address this problem, set $\alpha=\beta$. In this case $\lambda$ measures the ratio of psychological values of gains and losses at any magnitude. Only in this case does $\lambda$ have a readily interpretable meaning, as Kahneman and Tversky (1979) intended.

We have focussed on the logistic stochastic extension of CPT. But the same problem will occur with the probit stochastic extension more common in experimental economics (e.g., Carbone \& Hey, 2000). Here the cumulative standard normal distribution replaces the logistic function, with the standard deviation parameter taking the role of the sensitivity 
parameter $\theta$ we have discussed above.

\section{Conclusion}

Dimensional analysis is a method for keeping track of the units of parameters in the physical sciences. By applying this method to cumulative prospect theory we have explained why risk and sensitivity parameters are so strongly correlated. We have suggested a modification to the stochastic form of the theory which involves converting psychological values back into their certainty equivalents before converting their difference into a choice probability. This bug fix completely eliminates the correlation between risk and sensitivity parameters, and renders the parameters comparable and readily interpretable.

\section{References}

Broomell, S., \& Bhatia, S. (2014). Parameter recovery for decision modeling using choice data. Decision, 1, 252-274. doi: 10.1037/dec0000020

Busemeyer, J. R., \& Townsend, J. T. (1993). Decision field theory: A dynamic-cognitive approach to decision making in an uncertain environment. Psychological Review, 100, 432-459. doi: 10.1037/0033-295X.100.3.432

Carbone, E., \& Hey, J. D. (2000). Which error story is best? Journal of Risk and Uncertainty, 20, 161-176. doi: 10.1023/A:1007829024107

Fourier, J. (1822). Théorie analytique de la chaleur. Paris: Firmin Didot.

Gäechter, S., Johnson, E. J., \& Herrmann, A. (2007). Individual-level loss aversion in riskless and risky choices. SSRN Working Paper. Retrieved from https://ssrn. com/abstract $=1010597$

Glöckner, A., \& Betsch, T. (2008). Do people make decisions under risk based on ignorance? an empirical test of the priority heuristic against cumulative prospect theory. Organizational Behavior and Human Decision Processes, 107, 75-95. doi: 10.1016/j.obhdp.2008.02.003

Glöckner, A., \& Pachur, T. (2012). Cognitive models of risky choice: Parameter stability and predictive accuracy of prospect theory. Cognition, 123, 21-32. doi: 10.1016/j.cognition.2011.12.002

Holt, C. A., \& Laury, S. K. (2002). Risk aversion and incentive effects. American Economic Review, 92, 1644-1655. doi: 10.1257/000282802762024700

Kahneman, D., \& Tversky, A. (1979). Prospect theory: An analysis of decision under risk. Econometrica, 47, 263-291. doi: 10.2307/1914185

Navarro-Martinez, D. M., Loomes, G., Isoni, A., \& Butler, D. (2017). Boundedly rational expected utility theory. MPRA working Paper. Retrieved from https://mpra.ub.uni-muenchen.de/79893

Nilsson, H., Rieskamp, J., \& Wagenmakers, E.-J. (2011). Hierarchical bayesian parameter estimation for cumulative prospect theory. Journal of Mathematical Psychology, 55, 84-93. doi: 10.1016/j.jmp.2010.08.006

Pachur, T., \& Kellen, D. (2013). Modeling gain-loss asymmetries in risky choice: The critical role of probability weighting. Retrieved from https://mindmodeling.org/cogsci2013/papers/0571/paper0571.pdf 
Rieskamp, J. (2008). The probabilistic nature of preferential choice. Journal of Experimental Psychology: Learning, Memory, and Cognition, 34, 1446-1465. doi: $10.1037 / \mathrm{a} 0013646$

Scheibehenne, B., \& Pachur, T. (2015). Using Bayesian hierarchical parameter estimation to assess the generalizability of cognitive models of choice. Psychonomic Bulletin 86 Review, 22, 391-407. doi: 10.3758/s13423-014-0684-4

Stewart, N., Reimers, S., \& Harris, A. J. L. (2015). On the origin of utility, weighting, and discounting functions: How they get their shapes and how to change their shapes. Management Science, 61, 687-705. doi: 10.1287/mnsc.2013.1853

Stott, H. P. (2006). Cumulative prospect theory's functional menagerie. Journal of Risk and Uncertainty, 32, 101-130. doi: 10.1007/s11166-006-8289-6

Sutton, R., \& Barto, A. (1998). Reinforcement learning: An introduction. MIT Press.

Tversky, A., \& Kahneman, D. (1992). Advances in prospect theory: Cumulative representation of uncertainty. Journal of Risk and Uncertainty, 5, 297-323. doi: 10.1007/BF00122574

Wakker, P. P. (2010). Prospect theory: For risk and ambiguity. Cambridge University Press. 